\title{
Control of laminar-instability waves using a new technique
}

\author{
By H. W. LIEPMANN, G. L. BROWN† AND \\ D. M. NOSENCHUCK \\ Graduate Aeronautical Laboratories, \\ California Institute of Technology, Pasadena, CA 91125, U.S.A.
}

(Received 30 June 1981)

\begin{abstract}
A new technique using surface-film activators has been developed to induce and control laminar-instability waves by periodic heating. A flat plate was instrumented and installed in the GALCIT High-Speed Water Tunnel with flush-mounted surface heaters and probes. Extremely two-dimensional naturally occurring TolmienSchlichting (TS) waves were observed along with the subsequent formation of turbulent spots. Laminar-instability waves were then excited in a controlled fashion using the surface-mounted heaters. A preliminary experiment on cancellation of excited laminar-instability waves was carried out. Finally, turbulent spots were produced using amplitude-modulated bursts to form Gaussian TS wave packets. Flow visualization, along with wall shear measurements, was used to infer the velocity and vorticity field near the wall.
\end{abstract}

\section{Introduction}

The control of laminar-turbulent transition is still a major problem in applied fluid mechanics. As a necessary but not sufficient experiment, we are working on the control of laminar-instability waves and turbulent-spot formation in the boundary layer. The classical experiments by Schubauer \& Skramstad (1947) not only proved conclusively the correctness of laminar-instability theory but added a new approach to experimental fluid dynamics: the use of controlled disturbances as a flow diagnostic. The vibrating-ribbon technique that they introduced to excite vorticity waves has been used since then in any elaborate experiment on laminar boundary-layer and channel-flow instability transition.

The renewed strong interest in transition control, together with the realization that organized vortex structures play an important part in turbulent shear flows, leads quite naturally to an attempt to control boundary-or shear-layer disturbances actively. In principle, active control involves sensors, computers and activators. For the excitation study, and possible control of spanwise laminar-instability waves, the vibrating ribbon is not an ideal activator; its inherent inertia, for example, can be reduced only at the expense of increased fragility. Even at zero amplitude it presents a noticeable flow perturbation, and the introduction of any spanwise modulation is difficult. In addition, it is not easy to represent the vibrating ribbon as a source of disturbances in any theoretical study (Gaster 1965). In view of this fact, and guided in part by experience with second-sound studies in He II, we have used periodic heating of thin films as activators for boundary-layer oscillations (and shear-layer oscillations). At

$\dagger$ Present address: Aeronautics Research Laboratory (A.R.L.), Melbourne, Australia. 
zero current, these films do not disturb the flow at all. They can accommodate high frequencies and their length, width and shape can be chosen quite freely. They can be easily modulated both in time and spanwise co-ordinates.

The effect of surface heating on boundary-layer flow is readily seen from the boundary-layer momentum equation written in the form

$$
\rho u u_{x}+\rho v u_{y}+p_{x}-\mu_{T} T_{y} u_{y}=\mu u_{y y}
$$

Thus the effect of surface heating in a gas for which $\mu_{T}>0$ is such that $\left(u_{y y}\right)_{0}>0$, and hence must lead to an inflection point in $u(y)$, and therefore increased instability. Cooling has the opposite effect, and in liquids, for which usually $\mu_{T}<0$, heating stabilizes and cooling destabilizes the layer. The equivalence of the term $-\mu_{T} T_{y} u_{y}$ and of an effective $p_{x}$ has been used occasionally to demonstrate the relation between surface heating and stability (Liepmann \& Fila 1947). For example, heating the surface in a gas has roughly the same effect as an adverse pressure gradient.

In view of the interest in compliant surfaces, it is worth while to point out that similarly the term $\left(-\mu_{T} T_{y} u_{y}\right)_{0}$ can be locally interpreted as a non-vanishing $\rho v u_{y}$ at the surface. Consequently, in water, heating and cooling of the surface is roughly equivalent to suction and blowing or negative and positive surface displacements, respectively, with

$$
v_{0}=-\frac{1}{\rho} \frac{d \mu}{d T} \frac{\partial T}{\partial y}
$$

$v_{0} / U$ can be easily estimated. With the usual approximation of $u \sim y$ near the wall, one finds

$$
\frac{v_{0}}{U} \sim \frac{\operatorname{Pr}^{\frac{1}{3}}}{R_{x}^{\frac{1}{2}}}\left(\frac{x}{\xi}\right)^{\frac{1}{3}} \frac{d \log \mu}{d \log T} \frac{\Delta T}{T},
$$

where $x$ is the distance to the strip heater and $\xi$ is the heater width. For the conditions in the present study, this works out roughly to

$$
\left|\frac{v_{0}}{U}\right|=0 \cdot 0003 \Delta T
$$

where $\Delta T$ is the strip-heater overheat in ${ }^{\circ} \mathrm{C}$. For a gas, the corresponding number is negative and about twenty times smaller.

Periodic heating of a surface strip can thus be compared to small normal surface oscillations leading to a periodic redistribution of vorticity in the boundary layer. These surface elements, besides representing a much-improved technique for the controlled injection of laminar-instability waves, can be used in pairs or even arrays and, hence, can be phase-controlled to strengthen or weaken such waves. This fact was indeed the main reason for the development of the technique, which is an attempt to affect laminar instability and possibly transition by active control of TS waves. In addition, the heated-element technique is being applied to free shear layers in both water and air. The corresponding experiments will be reported elsewhere.

Finally, the experiments demonstrate that the heated-film technique can be used as a convenient, controllable, boundary-layer tripping device. 


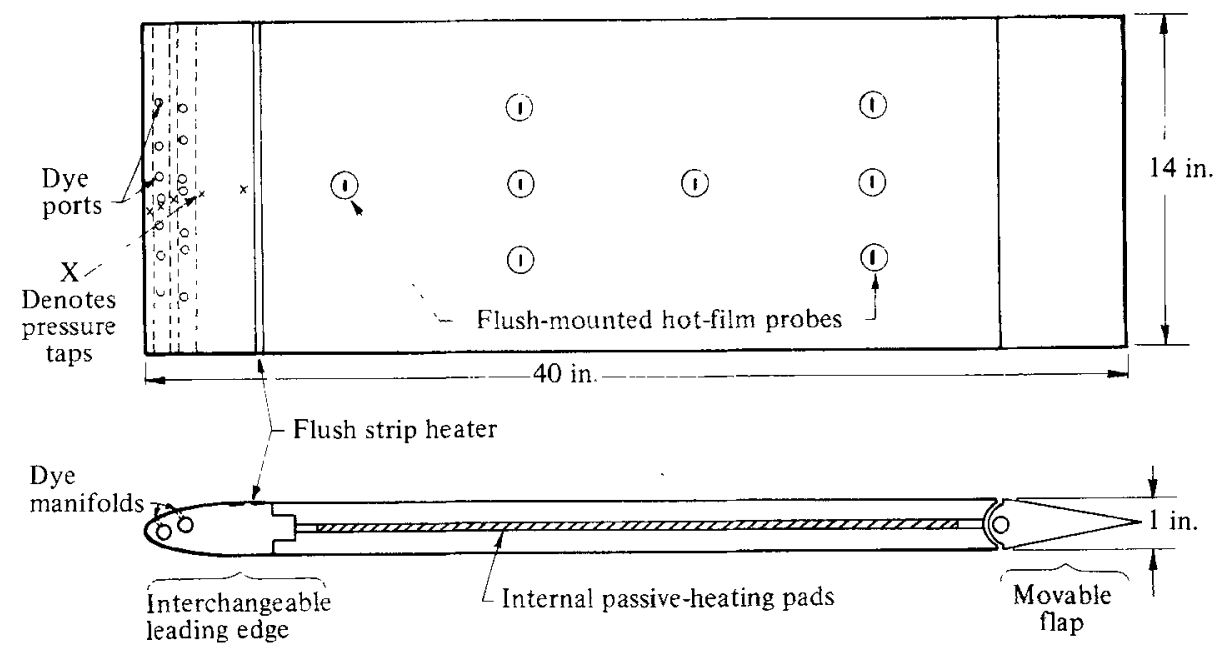

Figure 1. Flat-plate layout.

\section{Experimental set-up}

The boundary-layer experiments were carried out in the GALCIT high-speed water tunnel and, during its refurbishing, were continued in a small open flume of lesser flow quality. The test surface in both cases was a flat plate. In both cases, a set of hotfilm wall-shear probes was used to measure both wall shear and wall-shear fluctuations.

The active control strips consisted of $0.1 \mathrm{in}$. wide, $0.002 \mathrm{in}$. thick nichrome strips, flush mounted and coupled to an oscillator and amplifier.

The experimental set-up was complemented by a set of dye-injection orifices, which were used, for example, to study the lateral spread of the turbulent spots. Details of the tunnel and apparatus, as well as the digital data acquisition system, will be presented in a more complete forthcoming publication.

\section{Experimental considerations}

Any study of boundary-layer stability and transition requires a very high quality flow; the facility used in this experiment was the GALCIT high-speed water tunnel. It has a $90 \mathrm{ft}$ resorber section, of critical importance in the study of cavitation, which provides excellent isolation from the primary pump and very long settling time for the decay of large-scale disturbances. The contraction ratio is $20: 1$ and the free-stream turbulence level is below $0.04 \%$. (Details of the frequency content of the fluctuations at particular free-stream velocities are being obtained.) The test section is circular, and the flat plate was mounted across a diameter and aligned with the axis of the section. This had the advantage that the section could be simply rotated to make the plate either parallel with or perpendicular to the upstream turning vanes.

The plate itself is shown in figure 1. It is an upper and lower plate bolted together with an elliptic leading edge. Though not of importance in this work, it incorporates heating pads sandwiched between the two plates to permit an investigation of the effects of passive heating at high Reynolds number. The leading edge shown in the figure is a 4:1 ellipse, and is instrumented with pressure taps and two separate dye- 


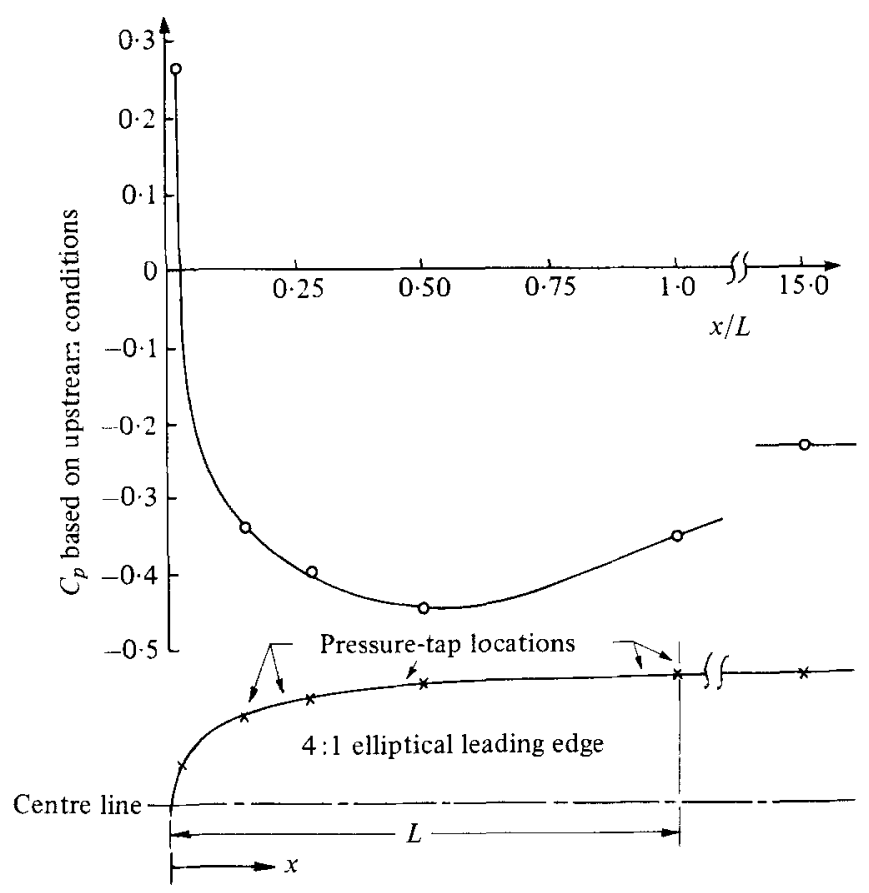

FIgure 2. Pressure distribution over flat-plate leading edge.

injection manifolds. The blockage ratio is $10 \%$, which assists in keeping the pressure overshoot on the ellipse to a low value. The actual pressure distribution is shown in figure 2, and for a Reynolds number of $2 \times 10^{6}$ based on plate length, the critical Reynolds number for this pressure distribution occurs at a centre-line distance from the leading edge of approximately $2 \mathrm{in}$. The dye-injection orifices are therefore upstream of this point, within the stable region of the boundary layer. The heating strip, on the other hand, is slightly downstream at an $R_{x}$ of $10^{5}$. For this first attempt to drive TS waves with local heating, the simplest heating strip was used, namely a $0.002 \mathrm{in}$. thick, $0.1 \mathrm{in}$. wide nichrome strip set into the leading edge and flush with the surface. Since the frequencies of the more unstable TS waves were typically $40 \mathrm{~Hz}$, this strip is easily shown to have an adequate time response. Assuming the wall shear is constant over the strip width and quasisteady heat transfer occurs, we estimate a thermal boundary-layer thickness $\theta$

$$
\theta=\text { const. } \times \delta\left(\frac{\xi}{x \operatorname{Pr}}\right)^{\frac{1}{3}}
$$

with a constant of approximately unity, where $x$ is the distance of the strip from the leading edge, $\xi$ its width and $\delta$ the local velocity boundary-layer thickness. For this case, the thermal thickness is approximately $15 \%$ of the boundary-layer thickness. This choice of width was a compromise between the depth of penetration within the boundary layer of the vorticity perturbation due to this thermal layer, power requirements to drive the heater, and mechanical considerations.

An array of eight hot-film wall-shear probes arranged as in figure 1 was used to measure time-resolved shearing stress. The data acquisition was synchronized with the TS wave excitation and with ciné photographs of dye streaks in the boundary layer. 


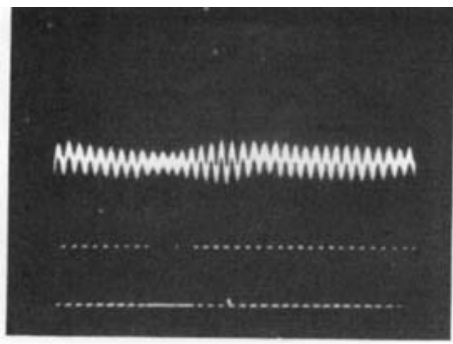

$R_{x}=2.5 \times 10^{5}$

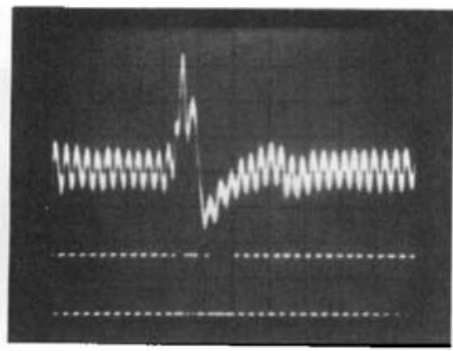

$4.0 \times 10^{5}$

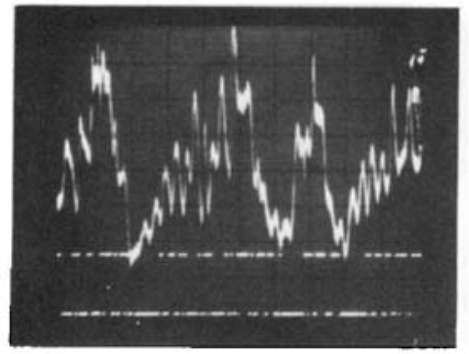

$6.5 \times 10^{5}$

(a)

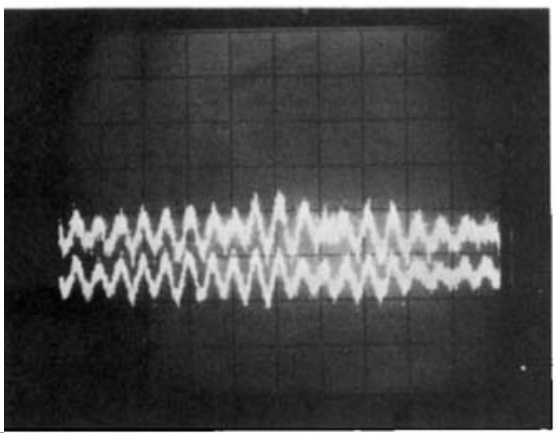

$50 \mathrm{~ms} / \mathrm{cm}$

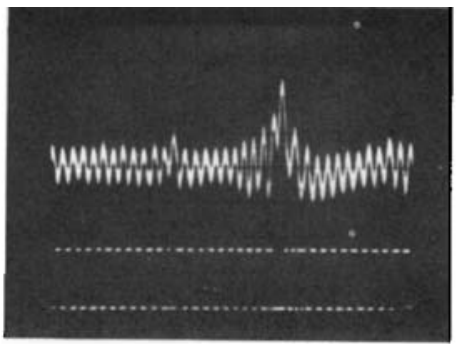

$3.5 \times 10^{5}$

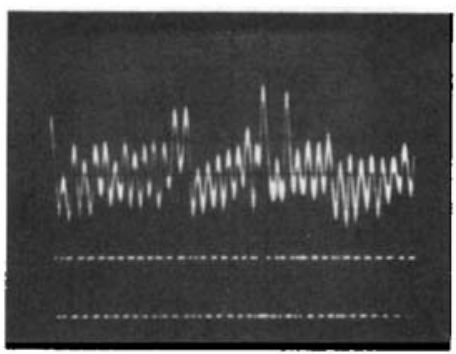

$4.5 \times 10^{5}$

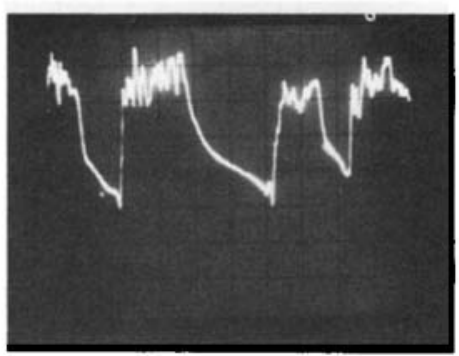

$9.0 \times 10^{5}$

(Vertical scale reduced in this photo)

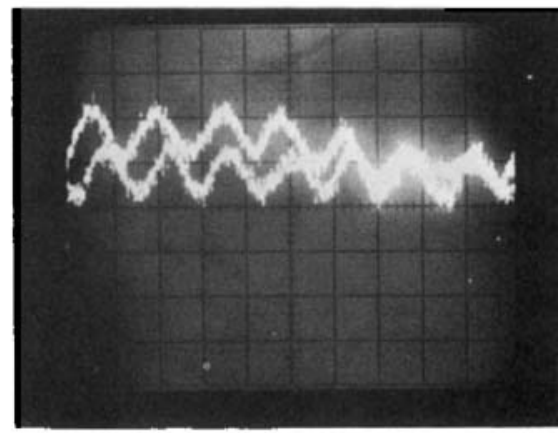

$20 \mathrm{~ms} / \mathrm{cm}$

Figure 3. (a) Natural transition: upper and lower traces show respectively wall shear and calibration marks; horizontal scale is $50 \mathrm{~ms} / \mathrm{div}$. (b) Two-dimensionality of naturally occurring TS waves; probe spacing $\simeq 5 \lambda ; R_{x}=5 \cdot 0 \times 10^{5}$. 

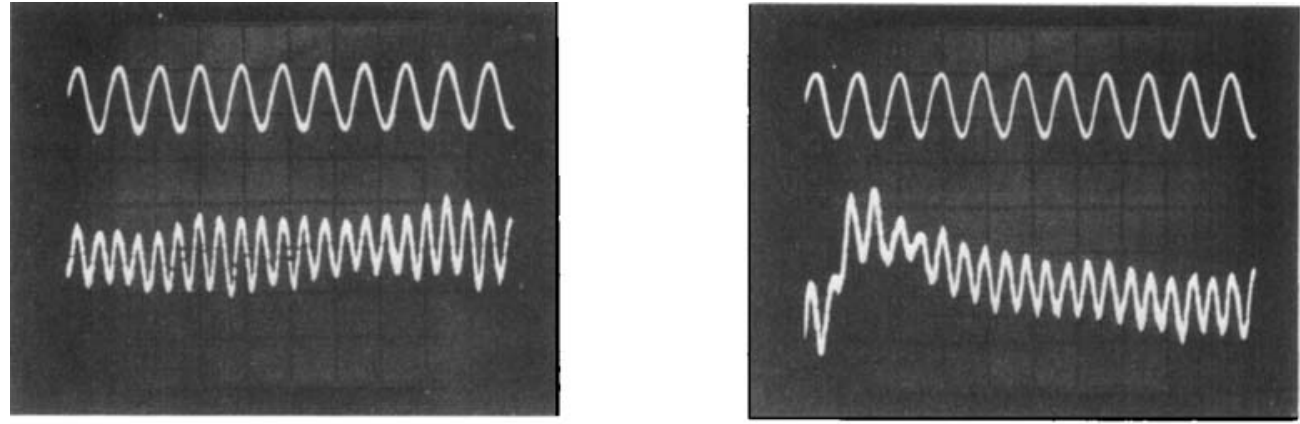

(a)
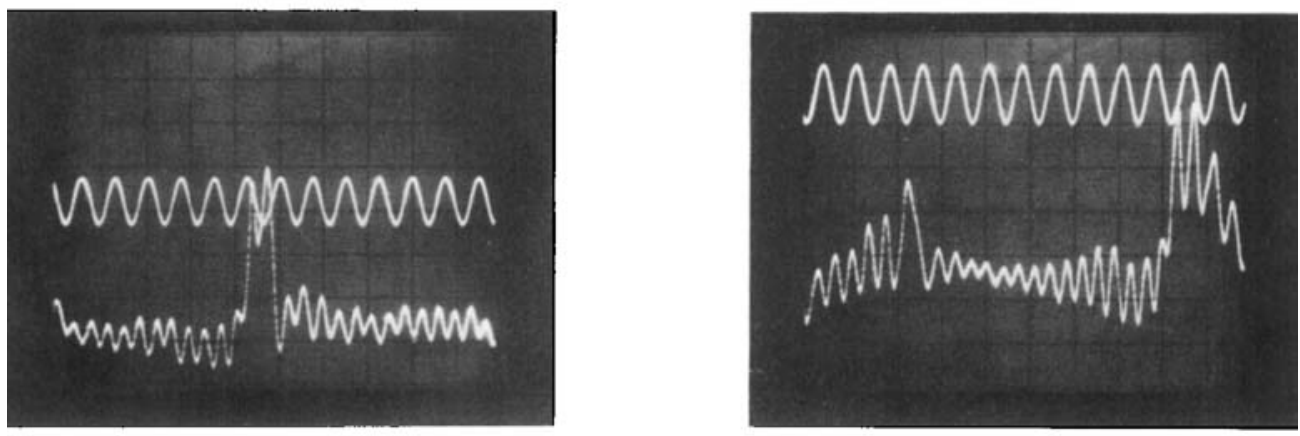

(b)

Fraure 4. Continuous forcing; $R_{x}=2.5 \times 10^{5}:$ (a) forcing frequency $26 \mathrm{~Hz}, 50 \mathrm{~ms} / \mathrm{div}$; (b) $40 \mathrm{~Hz}, 100 \mathrm{~ms} /$ div. Upper and lower traces show respectively input and measured shear.

The 16 dye streaks were grouped in pairs (hence the two-dye manifolds), the streaks of each pair being 0.05 in. apart (of the order of the boundary-layer thickness) in order to observe local features of the velocity field. Also in mind was the possibility of using two different and reacting dyes, as in recent shear-layer mixing experiments.

\section{Results}

\section{(i) Laminar instability}

Figure 3 shows the output of wall shear probes for naturally occurring transition. Figure $3(a)$ is remarkable for the initial uniformity and clarity of the spontaneously excited TS waves. The progression towards transition through sudden large increases in wall shear is less than a TS wavelength, and the emergence of turbulence spots is evident. Fully turbulent spots are apparent at $R_{x}=9 \cdot 0 \times 10^{5}$. Figure $3(b)$ demonstrates the two-dimensionality of these naturally occurring TS waves. The two probes were at the same distance from the leading edge and spaced 3.0 in. apart in the spanwise direction, or approximately five TS wavelengths. In the authors' experience, particularly in wind tunnels, the quality of this flow is quite exceptional, owing probably to the relatively very low acoustic fluctuation level in the tunnel, the extremely low tunnel-vibration level, and the poor coupling between the two for water.

Figures $4(a, b)$ show the success with which TS waves could be excited by periodic heating of the strip. The heater was directly coupled to the power amplifier with zero 

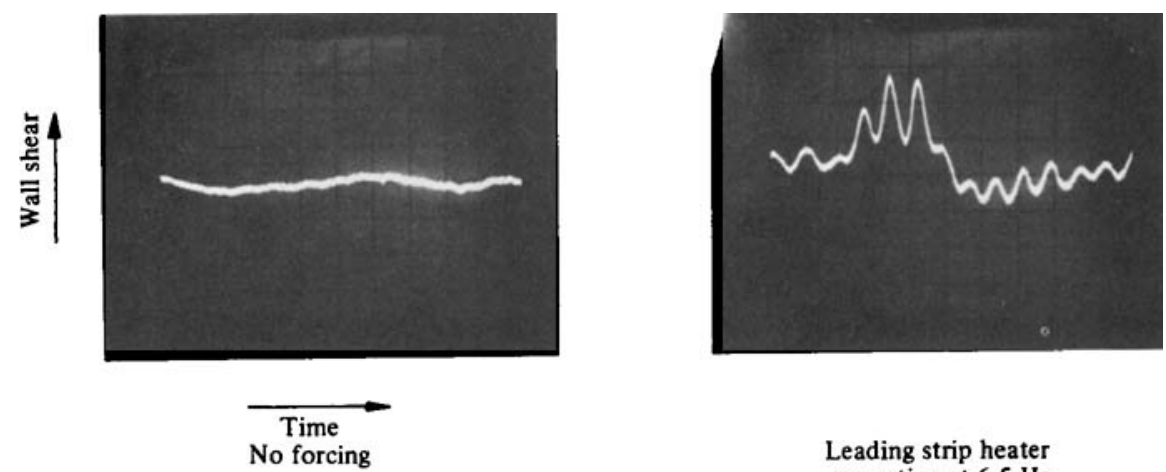

\section{No forcing}

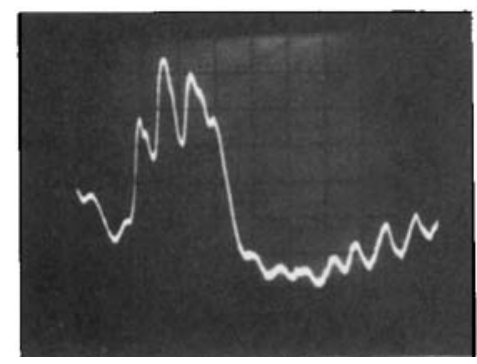

Trailing strip heater in phase with forced waves

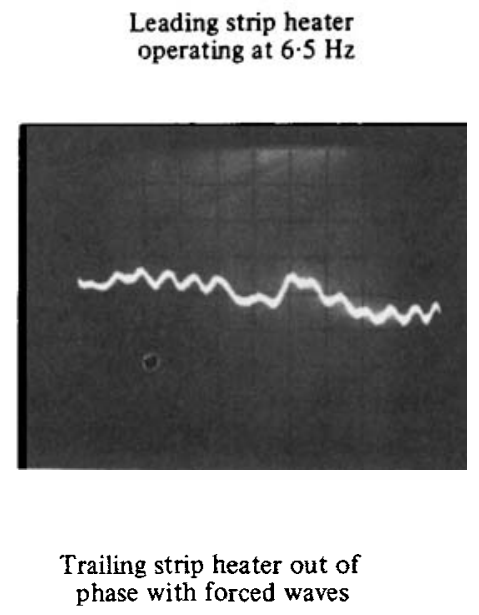

Figure 5. Continuous forcing with two strip heaters: $R_{x}=2 \cdot 2 \times 10^{5}, 200 \mathrm{~ms} / \mathrm{div}$.

offset so that the periodic heating was at twice the frequency of the electrical excitation. The upper trace in figure 4 is the heater voltage, the lower trace the output of a downstream wall-shear probe; the frequency doubling is clear.

In figure $4(b)$, the forcing frequency has been increased from that in figure $4(a)$, and at this higher frequency, stability theory shows that the amplitude of the disturbance grows more rapidly. It is evident that the evolution to three-dimensionality is more advanced in figure $4(b)$. (The typical heater power for these results was $15 \mathrm{~W}$, with a crudely estimated temperature rise $\simeq 3^{\circ} \mathrm{C}$. $\dagger^{\dagger}$

\section{(ii) Control of laminar instability}

The obvious and intriguing question that follows from this result is that if heating can be used to drive a TS wave, can a second heater be used to drive a second disturbance out of phase with the first to achieve cancellation of the first TS wave. If this were the case, then the control of naturally occurring TS waves would, in principle, follow, since the detection of the amplitude and phase of these waves is straightforward. To the extent that it is a linear problem, cancellation is clearly predicted (Gaster 1965). We were not able to put a second heater in the plate described before the high-speed

† The shear-stress measurements were not influenced by direct heating from the activator strips. This fact was verified by computation, measurements with a thermocouple and, most convincingly, by the lack of response to input frequencies corresponding to highly damped TS waves. 


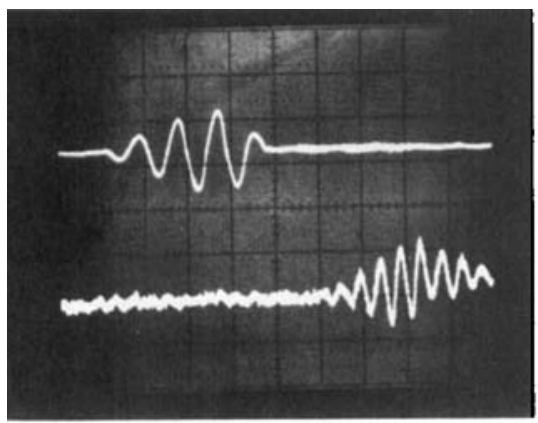

(a)

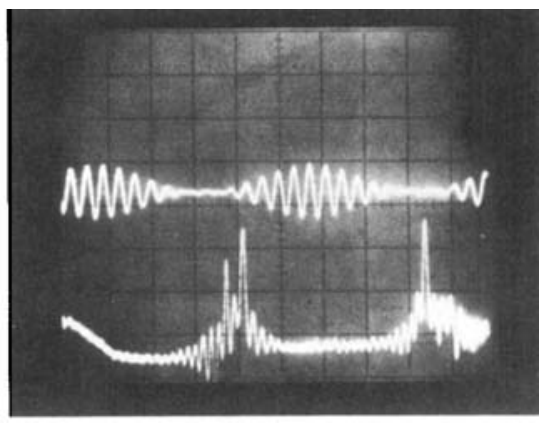

(b)

Fiaure 6. Amplitude-modulated forcing: (a) forcing (base) frequency $=22 \mathrm{~Hz}, R_{s}=2 \cdot 8 \times 10^{5}$, $50 \mathrm{~ms} / \mathrm{div}$; (b) $26 \mathrm{~Hz}, 5 \cdot 0 \times 10^{5}, 200 \mathrm{~ms} / \mathrm{div}$. Upper and lower traces show respectively input and measured wall shear.

tunnel was shut down for a major overhaul. As a result, we used a marginally satisfactory water channel with a much higher turbulence level and a different plate. Despite these difficulties, figure 5 shows that partial cancellation was achieved when two strip heaters were driven to produce out-of-phase disturbances and amplification when driven in phase. As the quality of the flow was relatively quite poor with a fully turbulent boundary layer at $R_{x} \simeq 6 \times 10^{5}$, we expect a more convincing outcome with more complete cancellation from the same experiment in the high-speed tunnel. We have just learned that similar cancellation experiments have been performed by Milling (1981).

\section{(iii) Transition}

To explore the transition process further, and at the same time demonstrate the flexibility of the dynamic-heating technique, we provided the heater with amplitudemodulated bursts to produce Gaussian wave packets of TS waves. Figure $6(a)$ shows the generated packet sensed at a later time by the wall-shear probe, compared with the voltage excitation. At a higher $R_{x}$, and with a larger number of waves in the packet, the typical rapid rise in shear within one TS wavelength is clear in figure $6(b)$, with the characteristics of a spot emerging (Emmons \& Bryson 1951).

With the ability to produce a small packet of TS waves, it was of interest to synchronize this packet with ciné photographs of the dye streaks in the boundary layer.

Figure 7 shows such a sequence in which a turbulent spot emerges from an initial Gaussian packet of TS waves generated by amplitude-modulated heating of the strip as in figure 6. The spanwise heating of the strip was uniform, so that initially the wares in the packet were two-dimensional. As the amplitude of the waves increased, threedimensionality developed, but the location of the emerging spot in the spanwise direction was random and varied with each TS wave packet. The development, as evident from the dye streaks, obviously requires TS waves of sufficient amplitude, as the typical downstream location at which it occurred could be mored upstream or downstream by changing the amplitude of the amplitude-modulated heater current. In figures $7(a, b)$, the packet of waves with their wave fronts perpendicular to the dye streaks is moving from left to right, and all dye streaks (many not shown) covering the central 8 in. of plate remained straight except for the central two pairs of the few pairs shown in the photograph. (As mentioned, in each pair the streaks are approximately 
0.05 in. apart.) These two pairs begin to show a waviness in figures $7(a, b)$ where the waviness has the same wavelength as that of the TS waves in the enveloping wave packet. This clearly indicates the beginning of a local departure from the initial two-dimensionality of the TS waves and mean flow, or more specifically, a local development of longitudinal vorticity. The symmetry of this development with respect to the dye streaks is fortuitous and, in fact, some ciné sequences suggest that the initial emergence of the local three-dimensionality does not always have spanwise symmetry, although, as a spot subsequently develops, symmetry is established. Figures $7(b-d)$ suggest that the development of this streamwise vorticity corresponds with a local warping of the initially parallel vortex lines, evidently in this case on a spanwise scale of order $\lambda$. (The rapid growth of the disturbance suggests that the diffusion of vorticity is relatively unimportant.) It is interesting that each pair of dye streaks become spread rapidly over distances significantly greater than $\delta$, as might be expected. since the induced motion of this increasing perturbation in the vorticity produces increasingly large $v$ - and $w$-components of velocity. Similarly, the dye becomes concentrated in the forward region of the disturbance because it has been lifted into a higher-velocity region of the boundary layer, thereby catching up with that released at an earlier time. Qualitatively the development of many features is strikingly similar to the numerical simulation of Leonard (1979).

In figures $7(e-g)$ the increasing longitudinal vorticity is particularly evident in the tail of the disturbance. There, the two dye streaks in each pair become increasingly spread in moving from the tail forward, indicating a large $c u / c x$. This suggests that the shear stress would correspondingly increase from the tail forward, which measurements confirm. It is very interesting to note in this sequence that the longitudinal vorticity increases and disturbances propagate laterally by an apparent destabilization process that seems very similar to the initial development of the dist urbance. That is, the neighbouring dye-streak pair marked $A$ shows a growing waviness in figures $7(d-g)$ not unlike the initial displacement of the central dye streaks of $7(a-c)$. This too corresponds with a warping of the vortex lines, initially perpendicular to the dye streaks, that is the development of longitudinal vorticity. This is a transversecontamination' process noted by Charters (1943) that accounts for the relatively rapid lateral spread of the emerging spot in this packet of TS waves. Gad-el-Hak, Blackwelder \& Riley (1980) observed a similar spanwise growth mechanism in the turbulent spot in a turbulent boundary layer, which, following Corrsin \& Kistler (1955), they term growth by destabilization. It is suggestive of a wave-like dist urbance propagating out along vortex lines.

These preliminary observations demonstrate some of the advantages of using dynamic heating to excite TS waves. The further experiments being carried out at present are intended to explore more fully the controllability of the initial TS waves as well as investigate further the departure from two-dimensionality by exploiting the simplicity with which controlled three-dimensionality can be superimposed.

The authors gratefully acknowledge support of this work by the Office of Naval Research under Contract N00014-78-C-0457. 


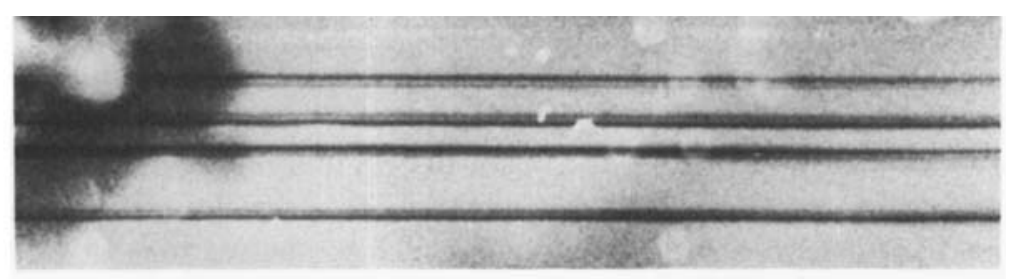

(a)
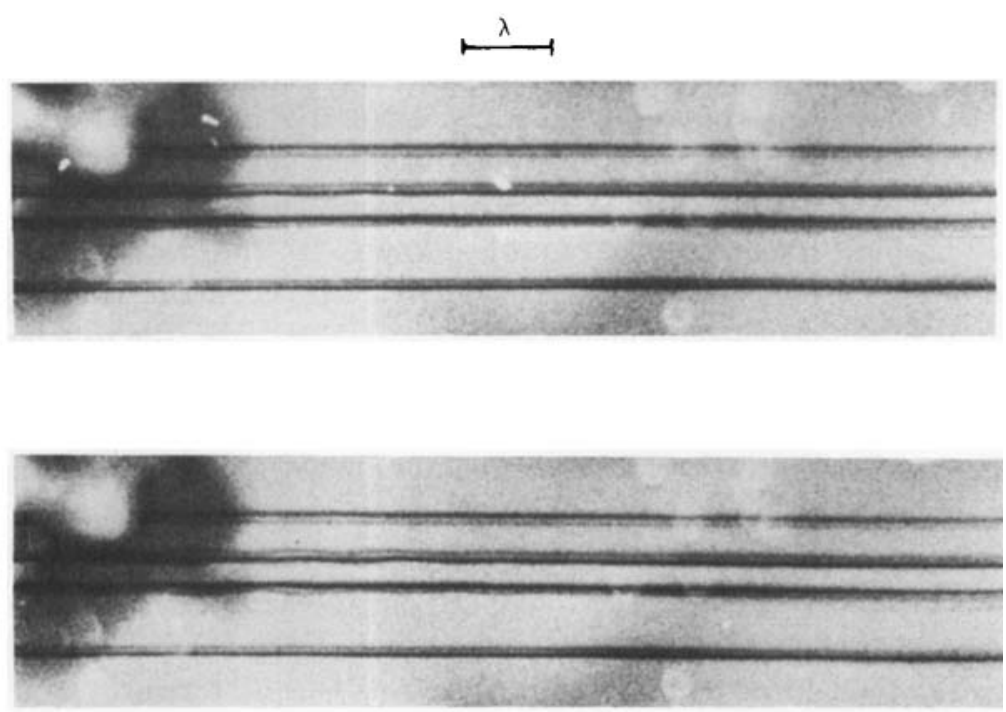

(b)

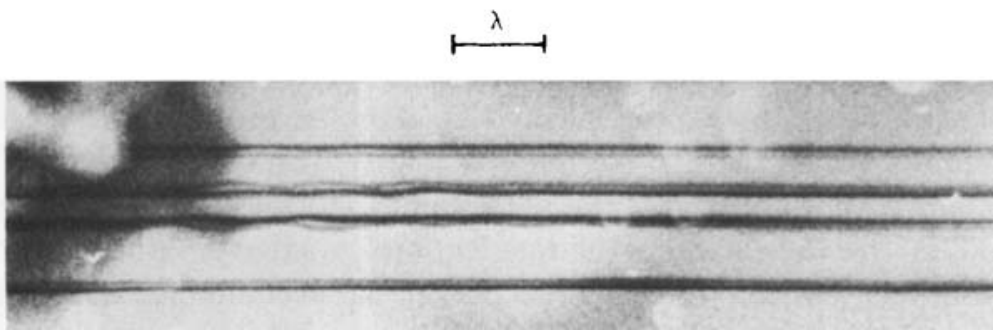

Frgure $7(a, b)$. Caption on p. 199. 


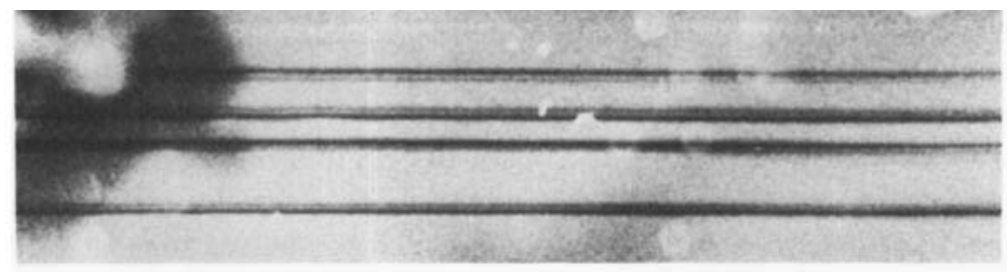

(a)
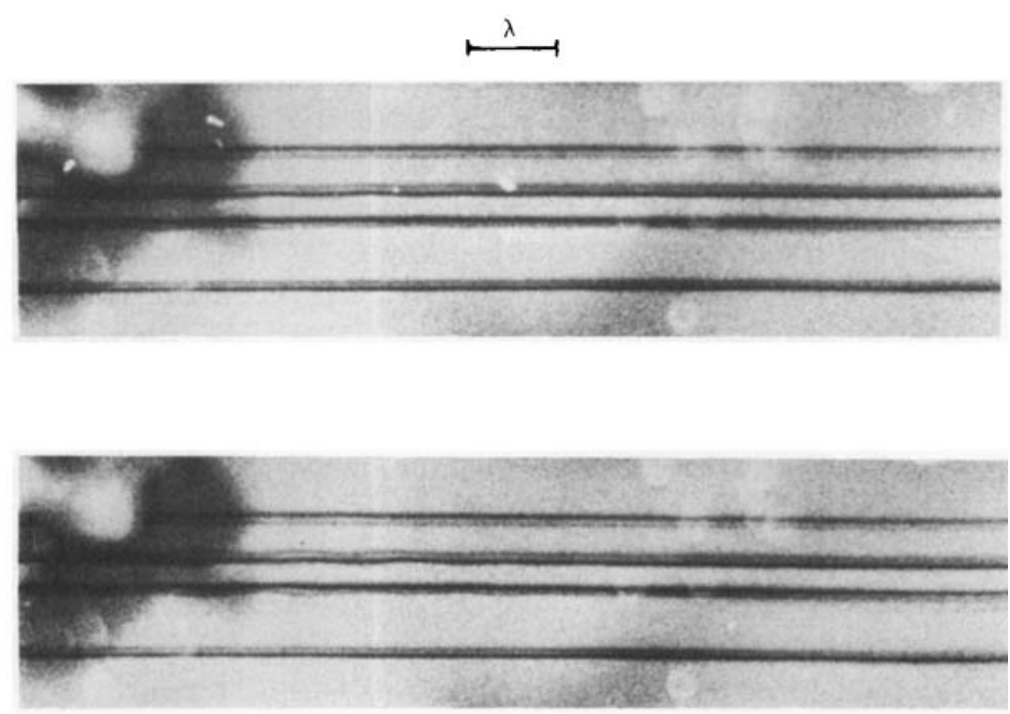

(b)
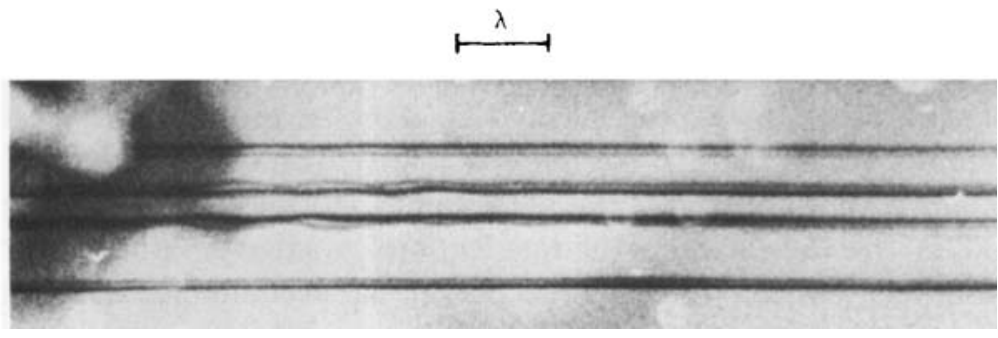

Fugure $7(c, d)$. Caption on p. 199. 


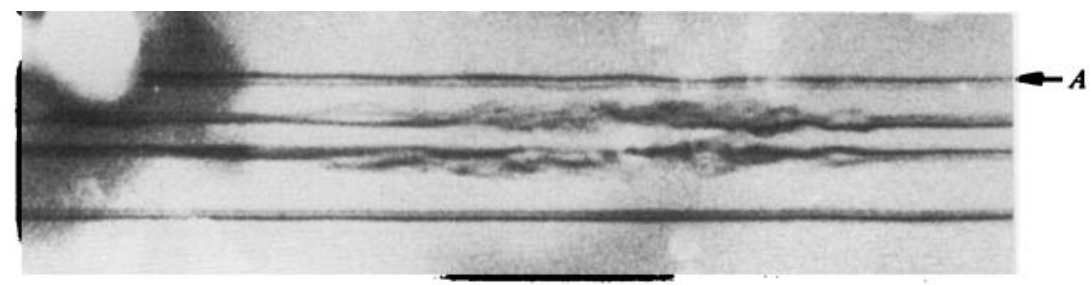

(e)
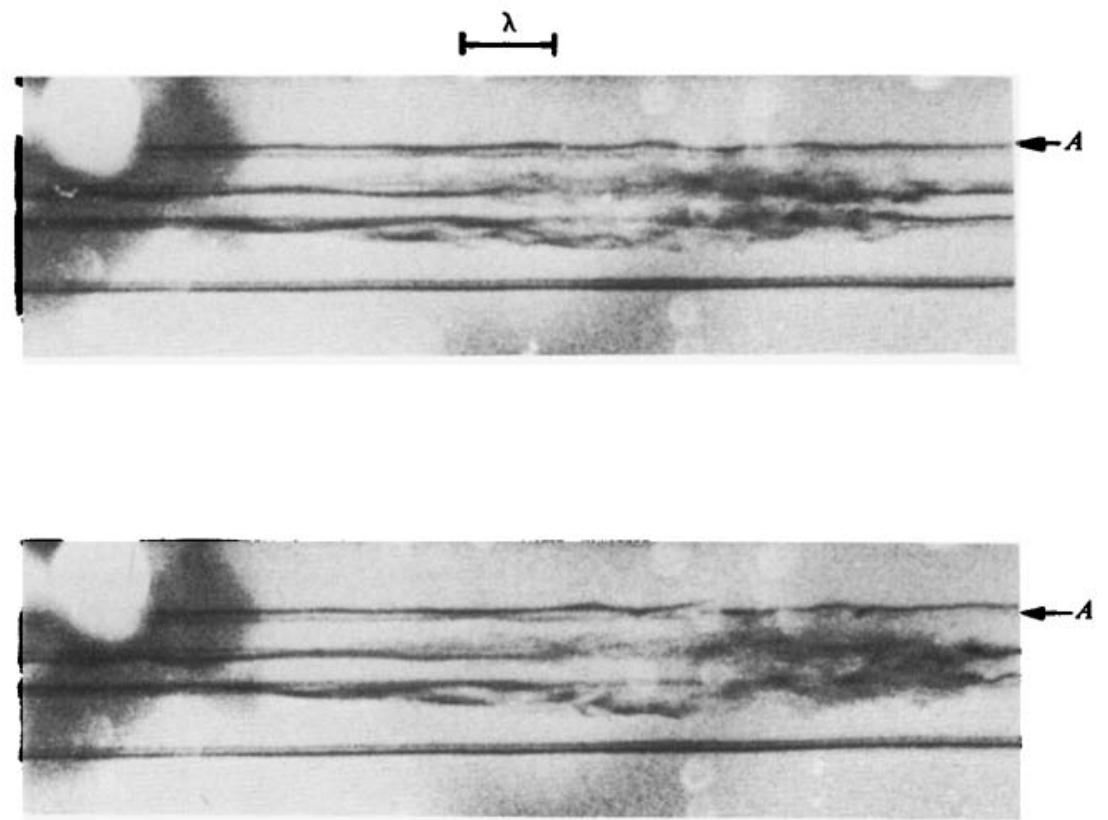

(f)

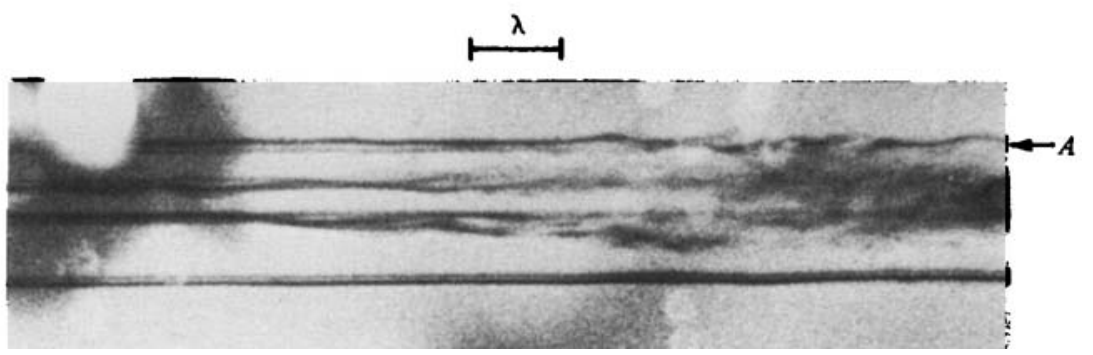

FIqURE $7(e, f)$. Caption on p. 199. 


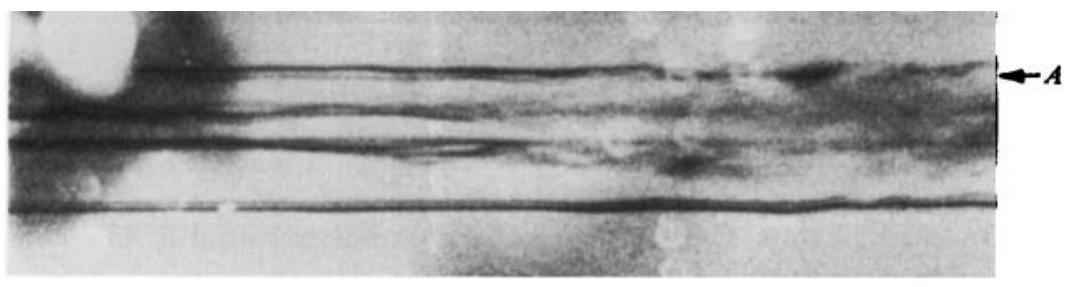

(g)
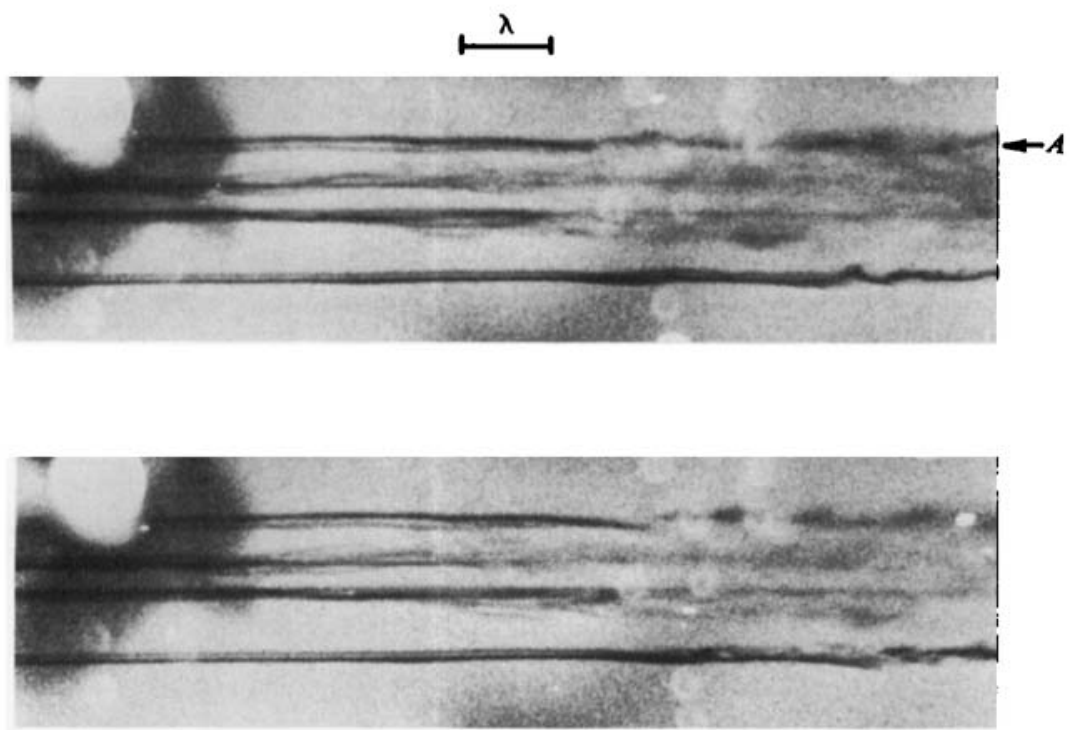

(h)

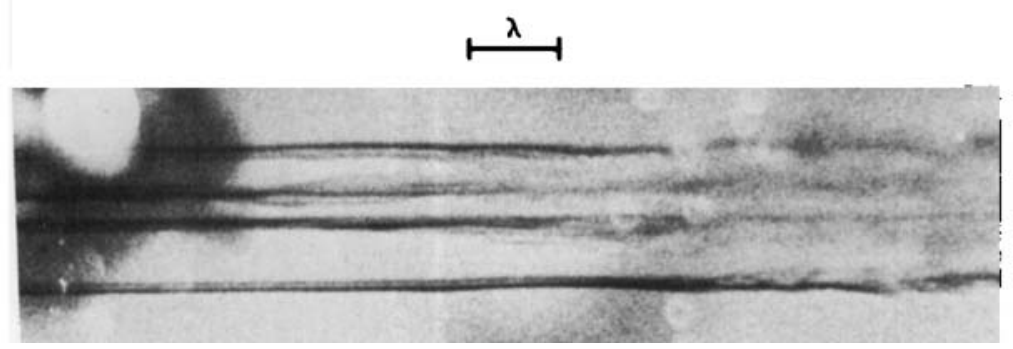

FiguRE 7. Turbulent-spot formation from TS wave packet. 


\section{REFERENCES}

Charters, A. C. 1943 Transition between laminar and turbulent flow by transverse contamination. NACA Tech. Note no. 891.

Corrsin, S. \& Kistier, A. L. 1955 Free stream boundaries of turbulent flows. NACA Rep. no. 1244.

Emmons, H. W. \& Bryson, A. E. 1951 The laminar turbulent transition in a boundary layer. Part 2. J. Aero. Sci. 18, 490-498.

GAD-EL-HAK, M., BLACKWELder, R. F. \& Rilex, J. J. 1980 A visual study of the growth and entrainment of turbulent spots. In Laminar-Turbulent Transition (ed. R. Eppler \& H. Fasel), pp. 297-310. Springer.

GASTER, M. 1965 On the generation of spacially growing waves in a boundary layer. J. Fluid Mech. 22, 433-441.

LiEPMANN, H. W. \& FirA, G. H. 1947 Investigation of effects of surface temperature and single roughness elements on boundary layer transition. NACA Rep. no. 890.

Milling, R. W. 1981 Tollmien-Schlichting wave cancellation. Phys. Fluids 24, 979-981.

Schubauer, G. B. \& Skramstad, H. K. 1947 Laminar boundary-layer oscillations and stability of laminar flow. J. Aero. Sci. 14, 68-78. 